\title{
Estudios Arqueofaunísticos en el Delta Superior del Paraná: el Sitio Los Tres Cerros 1 (Provincia de Entre Ríos, Argentina)
}

\author{
Zooarchaeological Research in the Upper Paraná Delta: Los Tres Cerros 1 Site (Entre \\ Ríos Province, Argentina)
}

Laura Bastourre ${ }^{\mathrm{i}}$

\begin{abstract}
RESUMEN
El sitio Los Tres Cerros I forma parte de una localidad arqueológica compuesta por tres estructuras monticulares localizadas en la isla Las Moras (departamento Victoria, Entre Ríos), datado entre 560 y 1030 años AP. El conjunto arqueofaunístico recuperado comprende una diversidad de taxones incluyendo mamíferos, aves, moluscos de río y abundantes peces. En este trabajo se presentan los resultados del análisis de los restos óseos asignados a mamíferos correspondientes a dos muestras localizadas en la cima y la base del montículo. Se aborda la diversidad taxonómica y anatómica del conjunto óseo y se relevan variables tafonómicas con el fin de comparar las actividades realizadas en ambos sectores del sitio. Los resultados permitieron establecer que los roedores, especialmente Myocastor coypus, predominan ampliamente en el conjunto de mamíferos y presentan claras evidencias de aprovechamiento antrópico. Las características particulares de las muestras de los dos sectores analizados (en cuanto a densidad de especímenes óseos, diversidad taxonómica y tamaño de los especímenes) permiten inferir una estructuración espacial del área de ocupación. En base a ello se infiere que la base del montículo funcionó como un área de basurero para el descarte de los desechos producidos por los grupos humanos que ocuparon el sitio a fines del Holoceno tardío.
\end{abstract}

Palabras clave: Delta Superior del Paraná, Zooarqueología, Descarte secundario

\begin{abstract}
Los Tres Cerros I site is part of an archaeological locality that comprises three mound structures located in Las Moras island (Victoria Departament, Entre Ríos Province) dated between 560 and 1030 years BP. Several taxa were identified in the zooarchaeological assemblage, including mammals, birds, freshwater mollusks and fishes. In this article the results of the analysis of bone remains assigned to mammals are presented. This sample belongs to two different sectors identified at the site: the top and the bottom of the mound structure. Taxonomical and anatomical diversity is explored and the taphonomic traces on the bones are assessed in order to compare the past activities carried out in both sectors. The results indicate that rodents, especially Myocastor coypus, are the most abundant taxa of the mammal assemblage and show clear evidence of anthropic processing. Differences between the samples recovered from both sectors (regarding density of bone specimens, taxonomic diversity and specimen size) indicate a spatial structuring of the occupied area. It follows that the
\end{abstract}

i División Arqueología, FCNyM, UNLP; Paseo del Bosque s/n, La Plata, CP 1900, Pcia. de Bs. As., Argentina. Correo-e:

laurabastourre@yahoo.com.ar

Recibido: 06-12-2012 Revisado: 09-08-2013 Aceptado: 12-12-2013 
bottom of the mound functioned as a midden area for the disposal of secondary refuse generated by the human groups that occupied the site during the Late Holocene.

Key words: Upper Paraná Delta, Zooarchaeology, Secondary refuse

\section{INTRODUCCIÓN}

En este trabajo se presentan los resultados del estudio arqueofaunístico llevado a cabo en el sitio Los Tres Cerros I (LTCI). Este sitio forma parte de una localidad arqueológica compuesta por tres montículos de tierra ("cerritos"), localizada en la isla Las Moras (departamento Victoria, Entre Ríos, Argentina). Las excavaciones arqueológicas llevadas a cabo hasta el momento cubrieron una superficie de $33 \mathrm{~m}^{2}$ y fueron efectuadas en dos sectores: la cima y la base del montículo (Figura I).

Los estudios geoarqueológicos y las II dataciones radiocarbónicas efectuadas en el sector de la cima del montículo indican que su formación ha sido el resultado de la acreción de estratos antrópicos durante una serie de eventos ocupacionales datados entre los $560 \pm 80$ y $1030 \pm 50$ años AP (Castiñeira et al. 20I2). Estos análisis permitieron delimitar dos sistemas depositacionales: uno natural, con dos unidades estratigráficas inferiores y un sistema antropogénico superior, donde pueden reconocerse tres capas principales. Las dos superiores presentan un alto contenido de materia orgánica, mientras que la capa más profunda está compuesta por lentes discontinuos de materia orgánica, carbón y sedimentos quemados (Castiñeira et al. 20I2, Politis et al. 20II). Los materiales arqueológicos recuperados incluyen abundantes restos faunísticos (óseos y malacológicos), numerosos tiestos cerámicos compatibles con la entidad GoyaMalabrigo, escasos restos líticos y algunos restos arqueobotánicos (Bonomo et al. 20ll I).

En la base del montículo se detectaron numerosos restos humanos $(\mathrm{NMI}=9$ ) en entierros tanto primarios como secundarios, individuales y múltiples así como restos óseos aislados. Dos fechados radiocarbónicos sobre restos óseos humanos de ca. 650 años AP indican la contemporaneidad de estas inhumaciones con al menos uno de los períodos de ocupación representados en la secuencia de la cima del montículo (Politis et al. 20I I, Scabuzzo y Ramos Van Rapp 2012). No es claro si los materiales faunísticos y cerámicos que se encontraron junto con los restos humanos son una mezcla de materiales descartados como producto de actividades domésticas o si corresponden a algún tipo de material asociado a entierros. Además, restos humanos aislados $(\mathrm{NMI}=\mathrm{l})$ con evidencias de termoalteración se recuperaron dentro de una estructura de fogón en la cima del montículo. Esto podría estar vinculado a algún tipo de ritual, incluyendo la cremación y la antropofagia (Politis et al. 20II). Finalmente, $\mathrm{LTCl}$ ha sido interpretado como un sitio de actividades múltiples, con un fuerte énfasis en el componente residencial y con ocupaciones relativamente estables (Bonomo et al. 20I I, Politis et al. 20I I).

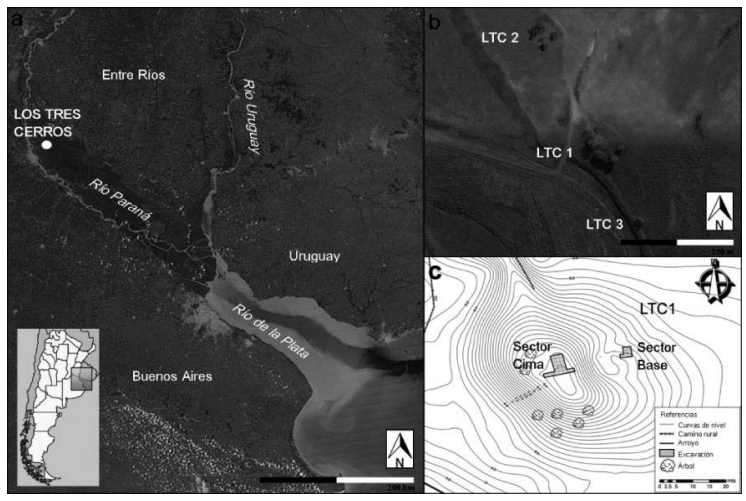

Figura 1: a) Ubicación de la localidad arqueológica Los Tres Cerros (LTC). b) Sitios 1, 2 y 3 de la localidad Los Tres Cerros (LTC1, LTC2 y LTC3). c) Ubicación de los sectores excavados en LTC1: cima y base de la estructura monticular; modificado de Castiñeira et al. 2013.

Figure 1: a) Location of Los Tres Cerros (LTC) archaeological locality. b) Sites 1, 2 and 3 of Los Tres Cerros locality (LTC1, LTC2 and LTC3). c) Location of the sectors excavated at LTC1: top and bottom of the monticular structure, modified from Castineira et al. 2013.

En este trabajo se presentan los resultados del análisis taxonómico y tafonómico de los restos óseos asignados a mamíferos correspondientes a dos muestras provenientes de los sectores mencionados anteriormente. Se pretende contribuir al conocimiento de las actividades de subsistencia de los grupos humanos que ocuparon LTCI en el pasado y a los procesos naturales y culturales que contribuyeron a la formación del sitio. Además, se 
comparan ambos conjuntos a través de diferentes variables (densidad de especímenes óseos, diversidad taxonómica y tamaño de los especímenes) con el objeto de detectar posibles diferencias en la estructuración del espacio ocupado.

\section{MATERIALESY MÉTODOS}

La muestra analizada comprende un total de II24 especímenes óseos correspondientes a mamíferos provenientes de las cuadrículas centrales $(4,5$ y 10$)$ del sector de la cima del sitio $(n=444)$ y de la base del montículo (cuadrículas II, 12, 28 y $29, \mathrm{n}=679$ ). Las cuadrículas centrales fueron analizadas en toda su extensión vertical, mientras que en el sector de la base se tomó una muestra de los niveles que presentaban una elevada abundancia de especímenes óseos (entre los 155 y $170 \mathrm{~cm}$ de profundidad). Cabe mencionar que en las cuadrículas consideradas se recuperaron gran cantidad de restos asignados a otros taxones $(n=10202)$, que no se abordan en detalle en este trabajo.

La cuantificación de los restos óseos se realizó a través de la aplicación de diferentes medidas de abundancia taxonómica y de partes esqueletarias: NISP, NMI, MNE, MAU y MAU\%. La evaluación de las variables tafonómicas se efectuó a través del examen macroscópico y con lupa binocular de la superficie cortical de los huesos. Se evaluó el grado de meteorización, la presencia/ausencia de distintas marcas de origen natural (roedores, raíces, carnívoros), la tinción por precipitaciones de manganeso, las alteraciones de origen antrópico (huellas de corte, raspado, machacado, percusión y aserrado perimetral), la termoalteración y los patrones de fractura siguiendo los lineamientos generales de diversos autores (Andrews 1990, Behrensmeyer, 1978, Fisher 1995, Gutiérrez 2004, Lyman 1994, Mengoni Goñalons 1999; entre otros).

\section{RESULTADOS}

\section{Abundancia taxonómica y anatómica}

Además de los mamíferos, las cuadrículas analizadas contienen una enorme abundancia de peces $(n=10167)$, correspondientes a Hoplias malabaricus (tararira), Leporinus obtusidens (boga), Cichlasoma facetum (chanchita) y a las familias Doradidae y Pimelodidae. Asimismo, se registraron restos de aves $(n=32)$ y reptiles $(n=3)$.

Como se observa en la Tabla I, el mamífero más representado en ambos conjuntos, tanto en NISP como en NMI es Myocastor coypus (coipo). En las cuadrículas centrales se estimó un NMI de 9 a partir del elemento más representado, los incisivos inferiores. En la base del montículo se observa una predominancia de hemimandíbulas a partir de las cuales se estimó un NMI de 10. En ambos conjuntos tanto los elementos del esqueleto axial como apendicular se encuentran bien representados. El siguiente taxón en abundancia es Hydrochoerus hydrochaeris (carpincho), que representa el $18 \%$ de la muestra correspondiente al sector de la base, mientras que se encuentra escasamente representado en las cuadrículas centrales (2\%). Las partes esqueletarias más frecuentes corresponden a elementos del miembro posterior (fémur y tibia) y elementos del cráneo. También se identificaron restos de Cavia aperea $(n=26)$, mientras que roedores de pequeño tamaño correspondientes a la subfamilia Sigmodontinae son muy poco frecuentes $(n=3)$. En conjunto, los roedores dan cuenta del $67 \%$ de la muestra de mamíferos.

Otros órdenes de mamíferos están representados por muy pocos especímenes, pero presentan claras evidencias de utilización antrópica. Es notable la escasa representación de los cérvidos, que hasta el momento sólo fueron identificados a través de un arpón elaborado sobre asta (Figura 2 g). Entre los especímenes asignados a cánidos (dentro de los que se identificó Dusicyon gimnocercus, $n=3$ ), un húmero distal presenta modificación por pulimiento. Por último, es necesario aclarar que la elevada proporción de especímenes de mamíferos indeterminados de las cuadrículas centrales corresponde en gran parte (45\%) a pequeños fragmentos de diáfisis de huesos largos de mamífero grande (> $50 \mathrm{~kg}$ ), sin atributos diagnósticos para su asignación taxonómica. Estos se hallan totalmente carbonizados y calcinados y provienen del nivel del fogón donde se hallaron los restos óseos humanos mencionados anteriormente. En este contexto, no se descarta que estos especímenes puedan corresponder a restos humanos, teniendo en cuenta además la presencia de pigmento sobre algunos de ellos. 


\begin{tabular}{|c|c|c|c|c|c|}
\hline \multirow{2}{*}{ Taxa } & \multicolumn{2}{|c|}{ Sector central } & \multicolumn{2}{|c|}{ Sector base } & \multirow{2}{*}{ NISP Tota } \\
\hline & NISP & $\mathrm{NMI}$ & NISP & NMI & \\
\hline Mammalia & $|5|$ & - & 215 & - & 366 \\
\hline Rodentia & 16 & - & 56 & - & 72 \\
\hline Myocastor coypus & 239 & 9 & 275 & 10 & 514 \\
\hline Caviidae & 5 & - & 2 & - & 7 \\
\hline Cavia aperea & 19 & 5 & 7 & I & 26 \\
\hline Hydrochoerus hydrochaeris & 9 & I & 122 & 4 & $|3|$ \\
\hline Sigmodontinae & 3 & - & - & - & 3 \\
\hline Cervidae & - & - & I & - & I \\
\hline Canidae & I & - & - & - & I \\
\hline Dusicyon gimnocercus & I & I & 2 & I & 3 \\
\hline Total & 444 & - & 680 & - & 1124 \\
\hline
\end{tabular}

Tabla 1: Valores de NISP y NMI para los taxones representados en LTC1.

Table 1: NISP and MNI values for the taxa represented at LTC1.

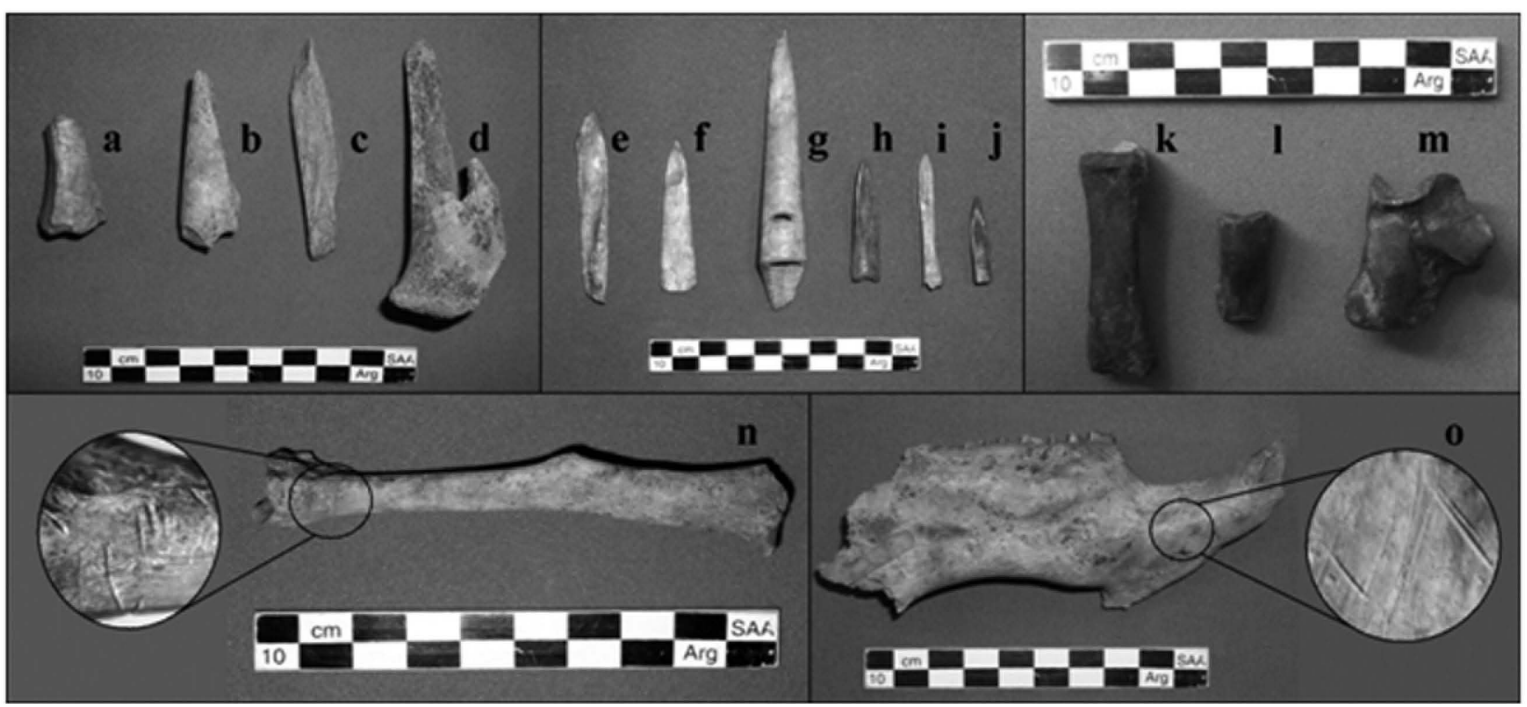

Figura 2: Modificaciones de origen antrópico: Aserrado perimetral sobre tibia de Myocastor Coypus (a). Fracturas helicoidales sobre hueso largo de mamífero indeterminado (b) y tibias de Hydrochoerus hydrochaeris (c y d). Algunos de los instrumentos recuperados en LTC1 (clasificación morfológica sensu Pérez Jimeno 2007): Punta acanalada sobre hueso largo (e); punta plana triangular sobre costilla

(f); arpón sobre asta (g) y puntas semiacanaladas sobre hueso largo indeterminado (h) y tibias de Myocastor coypus (i-j). Elementos termoalterados de Hydrochoerus hydrochaeris $(\mathrm{k}-\mathrm{m})$. Huellas de corte sobre tibia distal de Myocastor coypus (n) y hemimandíbula de Hydrochoerus hydrochaeris (o)

Figure 2: Bone modifications by humans: Saw marks on Myocastor Coypus tibia (a). Helical fractures of indetermined mammal long bone (b) and Hydrochoerus hydrochaeris tibias ( $c y d$ ). Bone tools sample from LTC1: fluted point made from a long bone (e); flat triangular point made from a rib (f); antler harpoon $(g)$ and half-fluted points made from an indetermined long bone $(h)$ and Myocastor coypus tibias ( $i-j)$. Hydrochoerus hydrochaeris thermoalterated bones $(k-m)$. Cut marks on Myocastor coypus distal tibia $(n)$ and Hydrochoerus hydrochaeris hemimandible (o). 


\section{Aspectos tafonómicos}

En ambos sectores la mayor parte de los especímenes presenta un estadio inicial de meteorización (estadio I; 35.5\%) o no se encuentra meteorizado (estadio 0; 33.7\%), indicando que los huesos no estuvieron expuestos durante un período prolongado antes de su sepultamiento. La depositación de óxido de manganeso es el proceso tafonómico de mayor incidencia, afectando al $67 \%$ de la muestra total, siendo ligeramente superior en el sector de la base (71\%) respecto a la cima del montículo (60\%). Esto se encuentra asociado a las propiedades del ambiente de depositación de los restos óseos, el cual está sometido a un régimen de inundaciones estacionales que inciden mayormente en los sectores topográficamente más deprimidos. Las marcas de roedores se registran sobre el $4.3 \%$ de los especímenes óseos. Los niveles con mayor frecuencia de estas marcas coinciden con aquellos donde se registró una elevada incidencia de bioturbación por crotovinas. Los carnívoros, aunque presentes en la muestra, no han dejado huellas sobre los restos óseos. Por otra parte, las marcas dendríticas producidas por raíces se registran en bajos porcentajes (3.7\%).

La mayoría de los restos óseos se encuentran fragmentados (74.5\%) y las fracturas se produjeron principalmente en estado seco (98.4\%). Fracturas helicoidales se registraron sobre huesos largos de carpincho y mamíferos indeterminados, algunas veces asociadas a lascados (Figura 2 b-d). Un caso de fractura transversal con aserrado perimetral sobre tibia proximal de coipo (Figura 2 a) puede posiblemente asociarse a la manufactura de los instrumentos que fueron elaborados sobre los extremos distales de estos elementos (ver Figura 2, i-j). Se detectaron huellas de corte sobre 14 de los especímenes óseos, asignados a Myocastor coypus, Hydrochoerus hydrochaeris y a mamíferos indeterminados. En el primer caso (Figura 2 n), se registran mayormente sobre diáfisis distal de tibia y húmero, y diáfisis proximal de ulna y radio y pueden estar vinculadas a actividades de desarticulación. En el caso del carpincho (Figura 2 o), se localizan sobre la hemimandíbula y la escápula, posiblemente relacionadas al cuereo y descarne respectivamente. Otras alteraciones antrópicas incluyen huellas de machacado y el pulimiento de la superficie de algunos huesos (un húmero de cánido y una ulna de carpincho). Finalmente, el $19.2 \%$ de los especímenes óseos presenta algún grado de termoalteración, predominando la carbonización (40\%) (Figura 2 k-m).

\section{DISCUSIÓN}

Los conjuntos analizados presentan claras diferencias entre sí en cuanto a abundancia absoluta, $\mathrm{NMl}$, densidad de materiales por $\mathrm{cm}^{3}$, abundancia taxonómica relativa y tamaño de los especímenes. En los niveles analizados en la base del montículo los valores de NISP y NMI son más elevados para la mayoría de los taxones, sobre todo para las especies con evidencia de aprovechamiento antrópico. Estas diferencias se tornan significativas al comparar la densidad de materiales. Teniendo en cuenta la totalidad de los restos óseos recuperados, las cuadrículas centrales del sector de la cima presentan una densidad promedio de 50.35 especímenes óseos por nivel/cuadrícula $\left(0.00 \mathrm{I}\right.$ hallazgos $\left./ \mathrm{cm}^{3}\right)$. Considerando la densidad de hallazgos asignados a mamíferos, se observan tres picos de concentración en los niveles 3 a 5,10 y 14 coincidiendo en parte con lo observado en peces (véase Politis et al. 20II). En el sector de la base del montículo la densidad promedio (1597.28 hallazgos por nivel/cuadrícula; 0.03 I hallazgos $/ \mathrm{cm}^{3}$ ) supera tanto a los valores generales como a los calculados para los picos de concentración en las cuadrículas centrales (Figura 3).

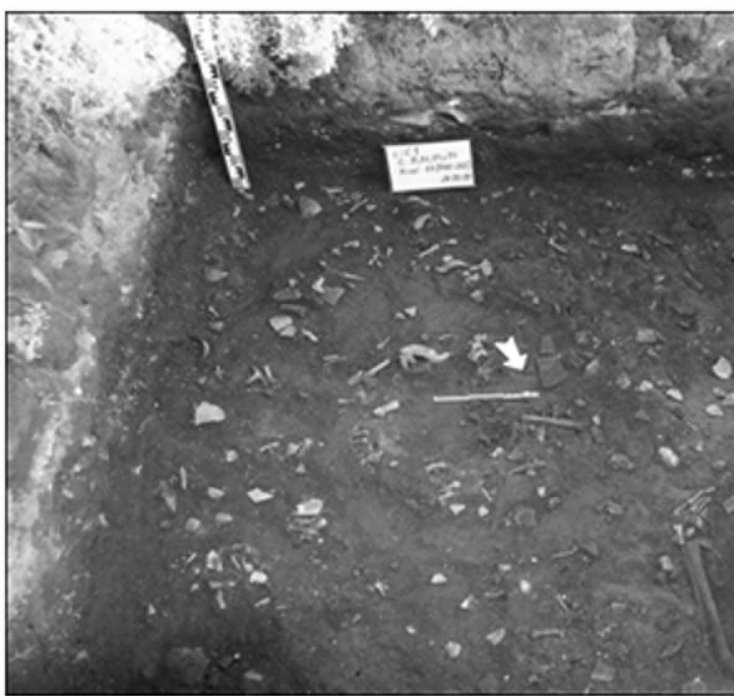

Figura 3: Área de basurero en Los Tres Cerros 1 (LTC1). Figure 3: Midden area at Los Tres Cerros 1 (LTC1). 
Asimismo, si consideramos las diferencias respecto a las fracciones de los especímenes, entendidas como porcentaje del elemento completo que se halla representado (Mengoni Goñalons 1999), observamos que en la base del montículo hay una mayor frecuencia de elementos completos o representados por una elevada fracción $(42 \%$ se hallan completos o representados por más del $70 \%$ del elemento). Otra diferencia significativa es la mayor abundancia relativa de Hydrochoerus hydrochaeris, roedor de gran porte, en la base del montículo. Estos dos factores se conjugan para generar un mayor tamaño de los especímenes óseos. Si bien esta variable está condicionada por el grado de fragmentación producido por procesos naturales y antrópicos, también puede vincularse con el manejo de los desechos dentro del área de ocupación. Así, puede esperarse que las áreas de actividad doméstica principales, en sitios con una ocupación relativamente estable, se mantengan limpias por la remoción de los desechos más grandes ( $\mathrm{O}^{\prime}$ Connell 1987, Schiffer 1983).

A la mayor densidad de especímenes óseos y el mayor tamaño de los mismos, se suma la presencia de partes esqueletarias con diferentes utilidades económicas en la base del montículo. Estos indicadores permiten sugerir que este sector funcionó, además de área de entierro, como un lugar para el descarte secundario de los restos óseos producidos a partir de las actividades domésticas llevadas a cabo en la cima del montículo.

\section{CONCLUSIONES}

Los principales mamíferos explotados en LTCl han sido los roedores Myocastor coypus e Hydrochoerus hydrochaeris, ambos con claras evidencias de aprovechamiento antrópico vinculadas tanto al consumo como a la manufactura de instrumentos. Esto se condice con otros sitios del Delta Superior donde Myocastor coypus ha sido identificado como uno de los principales recursos aprovechados (Bonomo et al. 20I I). Otros roedores más pequeños, probablemente sean intrusivos en el contexto arqueológico. Cánidos y cérvidos, aunque escasamente representados en LTCI, formaron parte de las especies aprovechadas para la confección de instrumental en hueso y asta.
Las diferencias entre los dos sectores analizados indican una estructuración del espacio en LTCI que podría relacionarse con la densidad y/o estabilidad de la ocupación humana del sitio. En la cima del montículo la mayoría de los materiales faunísticos probablemente sean producto del descarte primario residual, mientras que la elevada densidad de materiales en la base representaría un sector de basurero. Sin embargo, no se descarta que parte de los materiales faunísticos allí recuperados tengan algún significado en relación con los entierros humanos. Por último, es destacable que las diferencias encontradas respecto a la abundancia taxonómica relativa entre los dos sectores (fundamentalmente en el caso del carpincho) alertan sobre la necesidad de tener en cuenta la diversidad intrasitio a la hora de proponer hipótesis vinculadas a la subsistencia.

\section{BIBLIOGRAFÍA}

Andrews, P. 1990. Owls, Caves and Fossils. University of Chicago Press, Chicago.

Behrensmeyer, A.K. 1978. "Taphonomic and Ecologic Information from Bone Weathering”. Paleobiology 4 (2): I50-I62.

Bonomo, M., G. Politis y C. Gianotti. 2011. "Montículos, jerarquía social y horticultura en las sociedades indígenas del delta del río Paraná (Argentina)". Latin American Antiquity 22 (3): 297-333.

Castiñeira, C., A. Blasi, G. Politis, M. Bonomo, L. del Puerto, R. Huarte, J. Carbonari, F. Mari y F. García-Rodríguez. 20I2. "The origin and construction of pre-Hispanic mounds in the Upper Delta of the Paraná River (Argentina)". Archaeological and Anthropological Sciences. En prensa.

Castiñeira, C., A. Blasi, M. Bonomo, G. Politis y E. Apolinaire. 2013. "Modificación antrópica del paisaje durante el Holoceno tardío: las construcciones monticulares en el Delta Superior del río Paraná". Revista de la Asociación Geológica Argentina. En prensa.

Fisher, W. 1995."Bone Modifications in Zooarchaeology". Journal of Archaeological Method and Theory 2 (I): 7-68.

Gutiérrez, M.A. 2004. Análisis Tafonómicos en el Área Interserrana (Provincia de Buenos Aires). Tesis Doctoral inédita. Facultad de Ciencias Naturales y Museo, Universidad Nacional de La Plata, La Plata.

Lyman, R.L. 1994. "Quantitative units and terminology in zooarchaeology”. American Antiquity 59 ( I): 36-7I.

Mengoni Goñalons, G.L. 1999. Cazadores de Guanacos de la Estepa Patagónica. Colección Tesis doctorales.de la Sociedad Argentina de Antropología, Buenos Aires.

O'Connell, J.F. 1987. A"lyawara Site Structure and Its Archaeological Implications". American Antiquity 52 (I): 74-I08.

Pérez Jimeno, L. 2007. Investigaciones arqueológicas en el sector septentrional de la llanura aluvial del Paraná, 
margen santafesina. La variabilidad del registro arqueológico. Tesis Doctoral inédita. Facultad de Ciencias Naturales y Museo, Universidad Nacional de La Plata, La Plata.

Politis, G., M. Bonomo, C. Castiñeira y A. Blasi. 20II. "Archaeology of the Upper Delta of Paraná River (Argentina): Mound Construction and Anthropic Landscapes in the Los Tres Cerros locality". Quaternary International 245: 74-88.
Scabuzzo, M.C. Y A. Ramos Van Rapp. 2012 "Dificultades en la determinación de sexo y edad en restos humanos aislados y dispersos. El caso de Los Tres Cerros I (Victoria, Entre Ríos)". Ponencia presentada en el I Taller Nacional de Bioarqueología y Paleopatología. Facultad de Filosofía y Letras, Universidad de Buenos Aires, Argentina.

Schiffer, M. 1983. “Toward the identification of Formation Processes”. American Antiquity 48 (4): 675-706. 